KCL-MTH-99-22

LAPTH-735/99

hep-th/???????

March 19, 2018

\title{
Simplifications of four-point functions in $N=4$ supersymmetric Yang-Mills theory at two loops
}

\author{
B. Eden ${ }^{a}$, P.S. Howe ${ }^{a}$, C. Schubert ${ }^{b}$, E. Sokatchev ${ }^{b}$ and P.C. West ${ }^{a}$ \\ a Department of Mathematics, King's College, London, UK \\ ${ }^{b}$ Laboratoire d'Annecy-le-Vieux de Physique Théoriqu円 LAPTH, Chemin de Bellevue, B.P. 110, \\ F-74941 Annecy-le-Vieux, France
}

\begin{abstract}
The superconformal Ward identities combined with $N=2$ harmonic analyticity are used to evaluate two-loop four-point correlation functions of gauge-invariant operators in $D=4, N=4$ supersymmetric Yang-Mills theory in terms of the well-known one-loop box integral. The result is confirmed by a direct numerical computation.
\end{abstract}

\footnotetext{
${ }^{1}$ URA 1436 associée à l'Université de Savoie
} 
Supersymmetric Yang-Mills (SYM) theory with maximally extended $(N=4)$ supersymmetry in four dimensions has long been known to have some very interesting properties, in particular, it is ultra-violet finite and hence superconformally invariant quantum mechanically [1]. It also admits monopole solutions which fall into spin one multiplets [2] and which play a crucial rôle in Montonen-Olive duality [3, 4]. There has been a great deal of recent interest in this theory as a superconformal field theory stimulated by the Maldacena conjecture via which it is related, in a certain limit, to IIB supergravity on $A d S_{5} \times S^{5}$ [5]. In a previous paper [6] we calculated fourpoint functions of certain gauge-invariant operators in this theory at two loops in perturbation theory using $N=2$ harmonic superspace Feynman diagrams [7]. The result involves three functions, $A_{1}, A_{2}$ and $A_{3}$ which can be expressed in terms of differential operators acting on a scalar two-loop integral [2. The first two of these can be reduced quite straightforwardly to the well-known one-loop box integral but the third is much more complicated and in [6] we were unable to simplify it. As a consequence, we could not confirm the conformal invariance of the two-loop contribution to the four-point function. Furthermore, all three functions are needed to construct the full $N=4$ amplitude. In this note we show how this problem can be solved by using the superconformal Ward identities for harmonic-analytic operators in harmonic superspace. More precisely, we show that from the Ward identities combined with the dynamical requirement of harmonic analyticity follow differential constraints on the coefficient functions. Thus, $A_{3}$ is determined in terms of $A_{1}$ and $A_{2}$, so that it too reduces to a one-loop integral. The relation between the two- and one-loop integrals we have found is far from obvious, so in order to confirm it we have also performed a direct numerical computation.

In $N=2$ harmonic superspace $N=4$ Yang-Mills theory is formulated in terms of an $N=2$ SYM multiplet, described by a prepotential $V^{++}$, and a hypermultiplet, $q^{+}$, both of which take their values in the Lie algebra of the gauge group $S U\left(N_{c}\right)$. Both of these superfields are Grassmann-analytic (G-analytic), that is they depend on half of the odd coordinates. We recall that $N=2$ harmonic superspace [8] is the product of Minkowski superspace $M$ with the sphere $S^{2} \sim S U(2) / U(1)$, and that the harmonic fields are defined on $M \times S U(2)$ but are equivariant with respect to the action of the $U(1)$ isotropy group of the sphere. The coordinates of this superspace are $\left\{x^{\alpha \dot{\alpha}}, \theta_{i}^{\alpha}, \bar{\theta}^{\dot{\alpha} i}, u_{i}^{+}, u_{i}^{-}\right\}$, where the $u_{i}^{ \pm}$together make up an $S U(2)$ matrix. A superfield $F^{q}(x, \theta, u)$ which has $U(1)$ charge $q$ is called Grassmann analytic if it satisfies

$$
D_{\alpha}^{+} F^{q}:=u_{i}^{+} D_{\alpha}^{i} F^{q}=0, \quad \bar{D}_{\dot{\alpha}}^{+} F^{q}:=u_{i}^{+} \bar{D}_{\dot{\alpha}}^{i} F^{q}=0 .
$$

These constraints are easily solved in an appropriate superspace frame and amount to functional dependence on $\theta^{+}:=u_{i}^{+} \theta^{i}, \bar{\theta}^{+}:=u_{i}^{+} \bar{\theta}^{i}$ only (but not on $\theta^{-}, \bar{\theta}^{-}$). Thus, G-analyticity is just a kinematical constraint (similar to chirality in $N=1$ superspace).

On shell, the hypermultiplet superfield $q^{+}$has the dynamical property of harmonic analyticity (H-analyticity)

$$
D^{++} q^{+}=0
$$

in addition to the kinematical property of Grassmann analyticity. Here $D^{++}, D^{--}=\overline{D^{++}}$and $D_{o}$ are the standard derivatives on $S U(2)$ (left-invariant vector fields) which fulfill the algebraic relations of the associated Lie algebra. $D^{++}$essentially corresponds to $\bar{\partial}$ on the sphere in the sense that a field of positive charge $q$ which is annihilated by $D^{++}$corresponds precisely to a holomorphic section of the line bundle $\mathcal{O}(q)$ over $S^{2}$; its dependence on the harmonic variables

\footnotetext{
${ }^{2}$ The coefficient $A_{2}$ was independently calculated using $N=1$ superfields in [9].
} 
is thereby restricted to be polynomial. In terms of component fields, the superfield equation (2) eliminates the infinite tower of auxiliary fields originating from the harmonic expansion on $S^{2}$. In addition, it puts the remaining physical fields on shell, therefore it is the field equation of the $N=2$ hypermultiplet.

The operators we are interested in are the gauge-invariant bilinear combinations of the hypermultiplet, $\operatorname{tr}\left(q^{+} q^{+}\right), \operatorname{tr}\left(\widetilde{q}^{+} q^{+}\right)$and $\operatorname{tr}\left(\widetilde{q}^{+} \widetilde{q}^{+}\right)$, where tilde denotes a conjugation which combines complex conjugation with the antipodal map on the sphere. These operators arise as "components" of the $N=4$ supercurrent multiplet of operators. Precisely this multiplet corresponds to $D=5, N=8$ supergravity on an $A d S$ background in the Maldacena conjecture, when it is decomposed into $N=2$ superfields. In [6] we showed how it is possible to reconstruct the leading scalar term of the $N=4$ supercurrent four-point function from the leading terms of three $N=2$ hypermultiplet bilinear four-point functions. Given this leading term, the entire superfunction can be obtained by means of $N=4$ superconformal symmetry.

The hypermultiplet bilinears satisfy both the G- and H-analyticity conditions on shell. Provided, therefore, that we are allowed to use the equations of motion of the underlying hypermultiplets, $n$-point Green's functions of such operators will be both G- and H-analytic functions on the product of $n$ copies of harmonic superspace. This circumstance, combined with the requirement of superconformal invariance of the $N=4$ theory, yields constraints on the Green's functions which can be understood as arising from the fact that analytic superconformal invariants have poles on $S^{2}$ while the Green's functions themselves must be regular. It is precisely these constraints which will allow us to establish the relation among the three coefficient functions of the four-point amplitude.

We now summarise the results of reference [6] for the four-point function $\left\langle\widetilde{q}^{+} \widetilde{q}^{+}\left|q^{+} q^{+}\right| \widetilde{q}^{+} \widetilde{q}^{+} \mid q^{+} q^{+}\right\rangle$ (as explained in [6], the other possible combinations of hypermultiplet bilinears can either be obtained from this one by permutations or vanish). Its leading (scalar) term in an expansion in the odd coordinates (denoted by " $\left.\right|_{\theta=0}$ ") is given by

$$
\begin{aligned}
\left.\left\langle\widetilde{q}^{+} \widetilde{q} \bar{q}^{+} q^{+}\left|\widetilde{q}^{+} \widetilde{q}^{+}\right| q^{+} q^{+}\right\rangle\right|_{\theta=0} & \\
& =\left[(14)^{2}(23)^{2} A_{1}+(12)^{2}(34)^{2} A_{2}+(12)(23)(34)(41) A_{3}\right],
\end{aligned}
$$

where

$$
A_{1}=\frac{\left(\partial_{1}+\partial_{2}\right)^{2} f(1,2,3,4)}{x_{14}^{2} x_{23}^{2}}, \quad A_{2}=\frac{\left(\partial_{1}+\partial_{4}\right)^{2} f(1,4,2,3)}{x_{12}^{2} x_{34}^{2}}
$$

and

$$
\begin{gathered}
A_{3}=4 i \pi^{2} \frac{\left(x_{24}^{2}-x_{12}^{2}-x_{14}^{2}\right) g_{3}+\left(x_{13}^{2}-x_{12}^{2}-x_{23}^{2}\right) g_{4}+\left(x_{24}^{2}-x_{23}^{2}-x_{34}^{2}\right) g_{1}+\left(x_{13}^{2}-x_{14}^{2}-x_{34}^{2}\right) g_{2}}{x_{12}^{2} x_{14}^{2} x_{23}^{2} x_{34}^{2}} \\
+\frac{\left(\partial_{2}+\partial_{3}\right)^{2} f(1,2,3,4)}{x_{14}^{2} x_{23}^{2}}+\frac{\left(\partial_{1}+\partial_{2}\right)^{2} f(1,4,2,3)}{x_{12}^{2} x_{34}^{2}} .
\end{gathered}
$$

Here 


$$
f\left(x_{1}, x_{2}, x_{3}, x_{4}\right)=\int \frac{d^{4} x_{5} d^{4} x_{6}}{x_{15}^{2} x_{25}^{2} x_{36}^{2} x_{46}^{2} x_{56}^{2}}
$$

and

$$
g_{4}\left(x_{1}, x_{2}, x_{3}\right)=\int \frac{d^{4} x_{5}}{x_{15}^{2} x_{25}^{2} x_{35}^{2}}
$$

(and similarly for $g_{1}, g_{2}$ and $g_{3}$ ) are two- and one-loop scalar integrals, correspondingly. As usual $x_{i j}:=x_{i}-x_{j}$. The dependence of the Green's functions on the harmonic variables is polynomial (a corollary of $\mathrm{H}$-analyticity) and is given by factors such as $(14)^{2}(23)^{2}$ where the abbreviation

$$
(12):=\epsilon^{j i} u_{i}^{+}(1) u_{j}^{+}(2)
$$

has been employed.

The one-loop triangle integral $g_{4}(17)$ is well-known [10]. Following [11] we express it as

$$
g_{4}\left(x_{1}, x_{2}, x_{3}\right)=-\frac{i \pi^{2}}{x_{12}^{2}} \Phi^{(1)}\left(\frac{x_{23}^{2}}{x_{12}^{2}}, \frac{x_{31}^{2}}{x_{12}^{2}}\right)
$$

where

$$
\Phi^{(1)}(x, y)=\frac{1}{\lambda}\left\{2\left(\operatorname{Li}_{2}(-\rho x)+\operatorname{Li}_{2}(-\rho y)\right)+\ln \frac{y}{x} \ln \frac{1+\rho y}{1+\rho x}+\ln (\rho x) \ln (\rho y)+\frac{\pi^{2}}{3}\right\}
$$

and

$$
\lambda(x, y) \equiv \sqrt{(1-x-y)^{2}-4 x y}, \quad \rho(x, y) \equiv 2(1-x-y+\lambda)^{-1},
$$

$\mathrm{Li}_{2}$ denoting the Euler dilogarithm. The two-loop scalar integral function $f\left(x_{1}, x_{2}, x_{3}, x_{4}\right)$ is not yet known in explicit form. However, this is no impediment to the calculation of $A_{1}$ and $A_{2}$ since the combination of derivatives on $f$ appearing here can be straightforwardly reduced to a one-loop box integral by using translation invariance for one of the subintegrations. Doing this one finds [6]

$$
A_{1}=\frac{4 \pi^{4}}{x_{14}^{2} x_{23}^{2} x_{12}^{2} x_{34}^{2}} \Phi^{(1)}\left(\frac{x_{14}^{2} x_{23}^{2}}{x_{12}^{2} x_{34}^{2}}, \frac{x_{13}^{2} x_{24}^{2}}{x_{12}^{2} x_{34}^{2}}\right) .
$$

$A_{2}$ differs from $A_{1}$ only by the interchange $2 \leftrightarrow 4$. In contrast to the evaluation of $A_{1}$ and $A_{2}$ the evaluation of $A_{3}$ is a formidable problem; the only explicit information on $A_{3}$ obtained in [6] was the cancellation of the leading $\frac{1}{x_{14}^{2}}$ - pole in $A_{3}$ in the limit $x_{14} \rightarrow 0$.

In the following we shall use conformal supersymmetry and harmonic analyticity to obtain a constraint equation between the three coefficient functions $A_{1}, A_{2}, A_{3}$. By conformal invariance we can rewrite these in terms of conformal cross ratios as follows: 


$$
\begin{aligned}
& A_{1}=\frac{a(s, t)}{x_{14}^{4} x_{23}^{4}} \\
& A_{2}=\frac{b(s, t)}{x_{12}^{4} x_{34}^{4}} \\
& A_{3}=\frac{c(s, t)}{x_{12}^{2} x_{23}^{2} x_{34}^{2} x_{14}^{2}}
\end{aligned}
$$

where

$$
s=\frac{x_{14}^{2} x_{23}^{2}}{x_{12}^{2} x_{34}^{2}}, \quad t=\frac{x_{13}^{2} x_{24}^{2}}{x_{12}^{2} x_{34}^{2}} .
$$

The entire G-analytic superfield four-point function can then be constructed systematically as a power series in $\theta^{+}$and $\bar{\theta}^{+}$using the superconformal Ward identities [12]. In addition, the dynamical requirement of $\mathrm{H}$-analyticity at the $\theta^{+} \bar{\theta}^{+}$level leads to the following differential constraints on the coefficient functions $a, b, c$ : 3

$$
\begin{aligned}
\beta_{s} & =-t \alpha_{s}-t \alpha_{t}, \\
\beta_{t} & =s \alpha_{s}+(s-1) \alpha_{t}
\end{aligned}
$$

where $\alpha, \beta$ are determined from the linear equations

$$
\begin{aligned}
b & =\alpha+\frac{\beta}{t}+\frac{a}{s}, \\
c & =-\frac{s}{t} \beta+\frac{t-s-1}{s} a .
\end{aligned}
$$

One sees that only two of the three coefficients are restricted. The set of first-order partial differential equations (15) is equivalent to a single second-order equation (the compatibility condition for the system (15)), e.g., for $\alpha$ :

$$
s \alpha_{s s}+t \alpha_{t t}+(s+t-1) \alpha_{s t}+2\left(\alpha_{s}+\alpha_{t}\right)=0 .
$$

Given some boundary conditions the above equations allow one to completely fix $\alpha$ and $\beta$, thus leaving arbitrary only the coefficient $a$.

In this note we shall concentrate on applying this general relation at the two-loop level. Here we have some additional information which simplifies the partial differential equations (15). From the explicit graph calculation we already know that (see (12), (13))

\footnotetext{
${ }^{3}$ The result we quote here follows from the general properties of the correlation functions and should be valid to all orders in perturbation theory, as will be shown in detail elsewhere [12].
} 


$$
a=4 \pi^{4} s \Phi^{(1)}(s, t), \quad b=4 \pi^{4} \Phi^{(1)}(s, t) .
$$

In terms of $\alpha$ and $\beta$ this becomes $\beta=-t \alpha$, after which the equations (15) can be easily solved to yield the following general solution:

$$
\alpha=\frac{\mu}{s}, \quad \beta=-\mu \frac{t}{s}, \quad \mu=\text { const . }
$$

Combining eqs. (13), (16), (18) and (19) we find

$$
x_{12}^{2} x_{23}^{2} x_{34}^{2} x_{14}^{2} A_{3}=\mu-4 \pi^{4}(s-t+1) \Phi^{(1)}(s, t) .
$$

The constant $\mu$ is easily shown to be zero using the known behaviour [6] of $A_{3}$ and $\Phi^{(1)}$ in the limit $x_{14} \rightarrow 0$. Thus we arrive at the following expression for $A_{3}$ :

$$
A_{3}=4 \pi^{4} \frac{x_{13}^{2} x_{24}^{2}-x_{14}^{2} x_{23}^{2}-x_{12}^{2} x_{34}^{2}}{x_{12}^{4} x_{34}^{4} x_{23}^{2} x_{14}^{2}} \Phi^{(1)}\left(\frac{x_{14}^{2} x_{23}^{2}}{x_{12}^{2} x_{34}^{2}}, \frac{x_{13}^{2} x_{24}^{2}}{x_{12}^{2} x_{34}^{2}}\right) .
$$

By (5) this result can be expressed as a partial differential equation for $f$,

$$
\begin{aligned}
x_{12}^{2} x_{34}^{2}\left(\partial_{2}+\partial_{3}\right)^{2} f\left(x_{1}, x_{2},\right. & \left.x_{3}, x_{4}\right)+(1 \leftrightarrow 3)= \\
& 4 \pi^{4}\left[\frac{x_{13}^{2} x_{24}^{2}-x_{14}^{2} x_{23}^{2}-x_{12}^{2} x_{34}^{2}}{x_{12}^{2} x_{34}^{2}} \Phi^{(1)}\left(\frac{x_{14}^{2} x_{23}^{2}}{x_{12}^{2} x_{34}^{2}}, \frac{x_{13}^{2} x_{24}^{2}}{x_{12}^{2} x_{34}^{2}}\right)\right. \\
& +\frac{x_{12}^{2}+x_{14}^{2}-x_{24}^{2}}{x_{24}^{2}} \Phi^{(1)}\left(\frac{x_{12}^{2}}{x_{24}^{2}}, \frac{x_{14}^{2}}{x_{24}^{2}}\right)+\frac{x_{12}^{2}+x_{23}^{2}-x_{13}^{2}}{x_{13}^{2}} \Phi^{(1)}\left(\frac{x_{12}^{2}}{x_{13}^{2}}, \frac{x_{23}^{2}}{x_{13}^{2}}\right) \\
& \left.+\frac{x_{23}^{2}+x_{34}^{2}-x_{24}^{2}}{x_{24}^{2}} \Phi^{(1)}\left(\frac{x_{23}^{2}}{x_{24}^{2}}, \frac{x_{34}^{2}}{x_{24}^{2}}\right)+\frac{x_{14}^{2}+x_{34}^{2}-x_{13}^{2}}{x_{13}^{2}} \Phi^{(1)}\left(\frac{x_{14}^{2}}{x_{13}^{2}}, \frac{x_{34}^{2}}{x_{13}^{2}}\right)\right] .
\end{aligned}
$$

This equation is non-trivial, and we have not been able to show that it is satisfied analytically, since $f$ itself is not known in sufficiently explicit form. Instead, we have used MATHEMATICA to evaluate both sides of the equation numerically, and have verified that they agree for more than twenty choices of the parameters $x_{i j}^{2}$ (with three-digit precision). For the evaluation of $f$ we used a two-parameter integral representation [13] which relates the integral (6) to the sunset diagram with one massless line [14, 15, 16, 17]. On the right hand side the explicit formula (10) was employed.

Retrospectively, the knowledge that all three coefficient functions in (3) are related to each other, so that it is sufficient to determine one of them, can be used to drastically simplify the graph calculation carried out in [6]. Suppose that we wish to compute only the coefficient $A_{2}$. To this end we can identify two pairs of harmonic variables as follows,

$$
u_{1} \equiv u_{4}, \quad u_{2} \equiv u_{3}
$$

so that only the structure $(12)^{2}(34)^{2} \rightarrow(12)^{4}$ survives. Then among all the two-loop graphs considered in [6] only the following one is non-vanishing: 


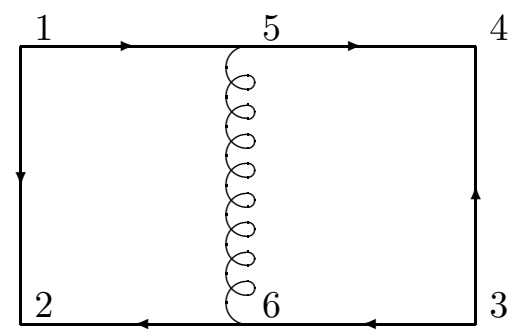

We recall that the matter propagators contain eight spinor derivatives, e.g.

1 5
$\frac{\left(D_{1}^{+}\right)^{4}\left(D_{5}^{+}\right)^{4} \delta^{8}\left(\theta_{1}-\theta_{5}\right)}{(15)^{3} x_{15}^{2}}$

whereas the SYM one has only four:

5

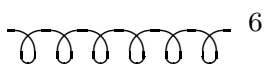

$\frac{\left(D_{5}^{+}\right)^{4} \delta^{8}\left(\theta_{5}-\theta_{6}\right)}{x_{56}^{2}} \delta\left(u_{5}, u_{6}\right)$

The vertices 5 and 6 are G-analytic, i.e. they involve the Grassmann integral measures $\int d^{4} \theta^{+}$. As usual in supergraph calculations, these measures have to be restored to the full superspace ones according to the identity $\int d^{4} \theta^{+}\left(D^{+}\right)^{4}=\int d^{8} \theta$. At the vertices 5 and 6 this can be done with the help of the spinor derivatives $\left(D_{5}^{+}\right)^{4}$ and $\left(D_{6}^{+}\right)^{4}$ from, e.g., the propagators $1-5$ and $6-2$. The remaining spinor derivatives $\left(D_{1}^{+}\right)^{4}$ and $\left(D_{2}^{+}\right)^{4}$ can then be integrated by parts onto the SYM line or the other two matter lines $5-4$ and 3-6. However, owing to the identification (23), the propagator $5-4$ has the same G-analyticity as $1-5$, and similarly for $3-6$ and $6-2$. Thus, all the spinor derivatives from $1-5$ and $6-2$ go onto the SYM line and we get twelve spinor derivatives on $\delta^{8}\left(\theta_{5}-\theta_{6}\right)$. Next, since the propagators $1-5$ and $6-2$ are now spinor derivative-free, the $\theta$ points 5 and 6 are identified with the external ones 1 and 2. Finally, we are only interested in the leading (scalar) term of the amplitude, so we can set $\theta_{1,2,3,4}=0$. This results in a D'Alembertian on the internal line $5-6$, which explains why the two-loop integral collapses to the one-loop integral $\Phi^{(1)}$.

To summarise, we have shown that, by combining the results of [6] with the constraints implied by superconformal invariance and H-analyticity, we are able to express the coefficient function $A_{3}$, which seemed to involve a "true" two-loop integration, and had so far defied all efforts at analytic evaluation, in terms of the functions $A_{1}, A_{2}$ which are easily expressed in terms of a single one-loop integral. This relation between $A_{1}, A_{2}$ and $A_{3}$ implies a non-trivial partial differential equation for the two-loop integral $f$ which we have confirmed numerically. We have also demonstrated that, once it is known that the calculation of the complete amplitude can be reduced to the calculation of either $A_{1}$ or $A_{2}$, this knowledge can be used to drastically simplify the Feynman diagram calculation. 
We should mention that neither the two-loop calculation performed in $[6]$ nor the analysis of the Ward identities presented here make any use of the full $N=4$ supersymmetry. In addition to $N=2$ supersymmetry, they rely only on finiteness (conformal invariance) which is known to be a property of a larger class of supersymmetric theories. At present we cannot say what specific rôle $N=4$ supersymmetry might play at higher orders of perturbation theory.

It should be emphasized that our final result is much simpler than one would a priori expect. Generically, a four-point two-loop integral such as the one encountered here is expected to contain, in addition to logarithms and dilogarithms of the kinematic variables, also tri- and quadrilogarithms. And indeed, the elusive function $f$ itself contains polylogarithms up to fourth order, as can be seen from the limiting case $x_{23}=0$, for which $f$ was obtained in closed form in [18]. It is tempting to speculate that the absence of tri- and quadrilogarithms in the two-loop result is just a glimpse of a universality property which would extend to all orders in perturbation theory for this amplitude, at least for large $N_{c}$. This seems even more plausible in view of the recent result that the same one-loop box function $\Phi^{(1)}$ appears also in the instanton contribution to four-point correlators in $N=4$ SYM theory [19]. The same simple dilogarithmic behaviour is found also for the spacetime dependence of the axion/dilaton amplitudes corresponding to the $N=4$ four-point function according to the Maldacena conjecture [20, 21, 22, 23].

Acknowledgements: We are indebted to A. Davydychev for repeatedly sharing with us his expertise on two-loop massless scalar integrals. We would also like to thank Z. Bern, O. Doré and K. Intriligator for helpful discussions, and D. Fliegner for computer support. This work was supported in part by the EU network on Integrability, non-perturbative effects, and symmetry in quantum field theory (FMRX-CT96-0012) and by the British-French scientific programme Alliance (project 98074).

\section{References}

[1] M. Sohnius and P.C. West, Phys. Lett. 100B (1981) 245; S. Mandelstam, Nucl. Phys. B213 (1983) 149; P.S. Howe, K.S. Stelle and P.K. Townsend, Nucl. Phys. B214 (1983) 519, Nucl. Phys. B236 (1984) 125; L. Brink, O. Lindgren and B. Nilsson Phys. Lett. 123B (1983) 323.

[2] H. Osborn, Phys. Lett 83B (1979) 321.

[3] C. Montonen and D. Olive, Phys. Lett 72B (1977) 117.

[4] A. Sen, Phys. Lett. 329B (1994) 217.

[5] J. Maldacena, Adv. Theor. Math. Phys. 2 (1998) 231, hep-th/9711200; S.S. Gubser, I.R. Klebanov and A.M. Polyakov, Phys. Lett. B428 (1998) 105, hep-th/9802109; E. Witten, Adv. Theor. Math. Phys. 2 (1998) 253, hep-th/9802150.

[6] B. Eden, P.S. Howe, C. Schubert, E. Sokatchev and P.C. West, KCL-MTH-98-58, LAPTH705/98, hep-th/9811172.

[7] A. Galperin, E. Ivanov, V. Ogievetsky, and E. Sokatchev, Class. Quantum Grav. 2 (1985) 601; 617; A.Galperin, Nguyen Anh Ky and E.Sokatchev, Mod. Phys. Lett. A 2. 
[8] A. Galperin, E. Ivanov, S. Kalitzin, V. Ogievetsky, and E. Sokatchev, Class.Quantum Grav. 1 (1984) 469.

[9] F. Gonzalez-Rey, I. Park and K. Schalm, Phys. Lett. B448 (1999) 37, hep-th/9811155.

[10] G. 't Hooft and M. Veltman, Nucl. Phys. B 153 (1979) 365.

[11] N.I. Ussyukina and A.I. Davydychev, Phys. Lett. B 298 (1993) 363.

[12] B. Eden, P.S. Howe, A. Pickering, E. Sokatchev and P.C. West, in preparation.

[13] A.I. Davydychev, private communication.

[14] S.C. Generalis, Doctoral thesis, OUT-4102-13 (Milton Keynes, UK, 1984).

[15] A. Djouadi, Nuovo Cim. 100A (1988) 357.

[16] J.B. Tausk, Doctoral thesis (Leiden, 1993).

[17] F.A. Berends and J.B. Tausk, Nucl. Phys. B 421 (1994) 456.

[18] N.I. Ussyukina and A.I. Davydychev, Phys. Lett. B 332 (1994) 159.

[19] M. Bianchi, M.B. Green, S. Kovacs and G. Rossi, JHEP 08 (1998) 013, hep-th/9807033.

[20] Hong-Liu and A.A. Tseytlin, Phys. Rev. D59 (1999) 086002, hep-th/9807097.

[21] D.Z. Freedman, S. Mathur, A. Matusis and L. Rastelli, Phys. Lett. B452 (1999) 61, hepth/9808006.

[22] E. D’Hoker, D.Z. Freedman and W. Skiba, Phys. Rev. D59 (1999) 045008, hep-th/9807098.

[23] E. D'Hoker, D.Z. Freedman, S.D. Mathur, A. Matusis and L. Rastelli, MIT-CTP-2843, UCLA/99/TEP/2, hep-th/9903196. 